\title{
HRT-Mediated Hypersensitive Response and Resistance to Turnip crinkle virus in Arabidopsis Does Not Require the Function of TIP, the Presumed Guardee Protein
}

\author{
Rae-Dong Jeong, ${ }^{1}$ A. C. Chandra-Shekara, ${ }^{1}$ Aardra Kachroo,, ${ }^{1}$ Daniel F. Klessig, ${ }^{2}$ and Pradeep Kachroo ${ }^{1}$ \\ ${ }^{1}$ Department of Plant Pathology, University of Kentucky, Lexington, KY 40514, U.S.A.; ${ }^{2}$ Boyce Thompson Institute \\ for Plant Research, Tower Road, Ithaca, NY 40053, U.S.A.
}

Submitted 29 April 2008. Accepted 6 June 2008.

\begin{abstract}
The Arabidopsis resistance protein HRT recognizes the Turnip crinkle virus (TCV) coat protein (CP) to induce a hypersensitive response $(\mathrm{HR})$ in the resistant ecotype Di17. The CP also interacts with a nuclear-targeted NAC family of host transcription factors, designated TIP (TCVinteracting protein). Because binding of CP to TIP prevents nuclear localization of TIP, it has been proposed that TIP serves as a guardee for HRT. Here, we have tested the requirement for TIP in $H R T$-mediated $H R$ and resistance by analyzing plants carrying knockout mutation in the TIP gene. Our results show that loss of TIP does not alter HR or resistance to TCV. Furthermore, the mutation in TIP neither impaired the salicylic acid-mediated induction of $H R T$ expression nor the enhanced resistance conferred by overexpression of $H R T$. Strikingly, the mutation in TIP resulted in increased replication of TCV and Cucumber mosaic virus, suggesting that TIP may play a role in basal resistance but is not required for HRT-mediated signaling.
\end{abstract}

Additional keywords: defense.

Plants have evolved some highly specific mechanisms to resist pathogens. The most studied of these involve the deployment of resistance $(\mathrm{R})$ proteins, which generally impart protection against specific races of pathogens carrying corresponding avirulence ( $A v r$ ) genes (gene-for-gene interactions) (Flor 1971). $\mathrm{R}$ proteins are believed to function as direct or indirect receptors for the appropriate Avr proteins. The tomato Pto and the rice Pita proteins were shown to interact with their cognate Avr proteins, AvrPto and Avr-Pita, respectively, in a yeast two-hybrid screen (Jia et al. 2000; Scofield et al. 1996; Tang et al. 1996), and the Arabidopsis RPS2 protein formed an in vivo complex with AvrRpt2 (Leister and Katagiri 2000). However, other R-Avr protein pairs have not yielded a detectable interaction (Nimchuk et al. 2000). Thus, it was suggested that R proteins "guard" other plant proteins that are targets of Avr proteins (Van der Biezen and Jones 1998; Innes 2004). Supporting this guard model, RIN4 was shown to physically interact with both the nucleotide binding-leucine rich repeat (NB-LRR) protein RPM1 and its

Corresponding author: P. Kachroo; E-mail: pk62@uky.edu; Telephone: +1.859.257.7445 ext. 80729; Fax: +1.859.323.1961.

Current address for A. C. Chandra-Shekara: DOW Agrosciences, Indianapolis, IN, U.S.A.

* The $\boldsymbol{e}$-Xtra logo stands for "electronic extra" and indicates that a supplemental figure is available online. avirulence factors AvrB and AvrRPM1 (Mackey et al. 2002). RIN4 also interacts with RPS2 and its avirulence factor AvrRpt2 (Axtell and Staskawicz 2003; Mackey et al. 2003). Thus, it was proposed that RIN4 is guarded by at least two different R proteins; modifications of RIN4 brought about by the various Avr proteins would lead to activation of the respective $\mathrm{R}$ proteins. Similarly, cleavage of protein kinase PBS1 by AvrPphB activates the R protein RPS5 (Shao et al. 2003). In addition, Avr2 inhibits the tomato Rcr3 protease required for $\mathrm{Cf}-2-$ mediated resistance (Rooney et al. 2005). These data strongly support an indirect mechanism of pathogen recognition by a majority of NB-LRR proteins.

Downstream of the recognition event, the signals activated by various Arabidopsis $\mathrm{R}$ proteins appear to converge into a small number of pathways (Kachroo et al. 2006; Parker et al. 1996). The pathway activated by toll interleukin 1 receptorNB-LRR proteins generally requires the EDS1 gene (Parker et al. 1996), while that activated by most coiled coil (CC)-NBLRR proteins requires the NDRl gene (Century et al. 1995). However, several CC-NB-LRR $R$ genes, including $R P P 8$, $R P P 13-N d$, and $H R T$, as well as $R P P 7$, signal resistance via one or more pathways that are independent of NDRl (BittnerEddy and Beynon et al. 2001; Chandra-Shekara et al. 2004; McDowell et al. 2000). Strikingly, the CC-NB-LRR gene $H R T$, which confers hypersensitive response (HR) and resistance to Turnip crinkle virus (TCV), is dependent on EDS1 (ChandraShekara et al. 2004).

Resistance to TCV is also dependent on EDS5, PAD4, and SID2 genes of the salicylic acid (SA) pathway but does not require $N P R 1, R A R 1, S G T 1$, or the defense hormones jasmonic acid or ethylene (Chandra-Shekara et al. 2004; Kachroo et al. 2000). Exogenous application of SA or the SA analog benzo $(1,2,3)$ thiadiazole-7-carbothioic acid $(\mathrm{BTH})$ restores resistance to TCV in SA-deficient Di-17 plants containing the $e d s 1$, eds5, or sid2 mutations. However, exogenous application of SA or BTH does not confer enhanced resistance in plants lacking HRT or PAD4 (Chandra-Shekara et al. 2004). In contrast to resistance, TCV-induced $\mathrm{HR}$ and $P R-1$ gene expression are not affected by mutations in the SA pathway, suggesting that these phenotypes are independent of SA.

In addition to $H R T$, resistance to TCV requires a recessive allele at a second locus, designated rrt (regulates resistance to TCV) (Chandra-Shekara et al. 2004, 2006; Kachroo et al. 2000). Exogenous application of SA or high endogenous levels of SA can overcome a requirement for rrt and confer resistance to TCV in an HRT-dependent manner (Chandra-Shekara et al. 2004, 2006). Transgenic overexpression of $H R T$ can also overcome a requirement for $r r t$ but is not associated with an 
increase in endogenous SA (Chandra-Shekara et al. 2004; Cooley et al. 2000).

HR to TCV is initiated upon direct or indirect interaction between HRT and viral coat protein (CP) (Cooley et al. 2000). TCV CP, which acts as an avirulence factor (Zhao et al. 2000), also interacts with a protein belonging to the NAC family of transcription activators, which is designated TIP (TCV-interacting protein) (Ren et al. 2000, 2005). Furthermore, transient coexpression of TIP and CP in tobacco cells has shown that TCV CP prevents nuclear localization of TIP (Ren et al. 2005). These observations have prompted the suggestion that HRT guards TIP and is activated when CP retains TIP in the cytosolic compartment (Ren et al. 2005; Soosaar et al. 2005).

In this report, we have analyzed the requirement of TIP for basal resistance to TCV and Cucumber mosaic virus (CMV) and for HRT-mediated HR and resistance to TCV. We show that TIP is required for basal resistance to CMV but not for HRT-mediated HR and resistance to TCV. Furthermore, absence of TIP does not affect the SA-mediated induction of $H R T$ or alter any of the phenotypes associated with overexpression of $H R T$. Our results suggest that the interaction between $\mathrm{CP}$ and TIP does not govern downstream signaling leading to HR formation and resistance.

\section{RESULTS}

A mutation in TIP does not abolish HR or resistance to TCV.

To study the role of TIP in HRT-mediated signaling, we examined the SALK insertional database for lines that carry TDNA insertion within TIP. One line was obtained in which the T-DNA was inserted 15 bases upstream of the translational start site (Fig. 1A). Plants homozygous for the T-DNA insertion were obtained and analyzed for TIP expression. At least five different homozygous lines were analyzed, and none of these showed any detectable expression of TIP (Fig. 1B). The TIP knock-out (KO) plants exhibited a normal morphology (Fig. 1C), which was consistent with basal levels of expression of $P R-1$ and absence of microscopic cell death in tip plants (Fig. 1D and data not shown).

Since retention of TIP in the cytosol has been proposed to induce HRT-mediated signaling (Ren et al. 2005), we analyzed the role of TIP in an HRT background. TCV resistant Di-17 (Dijon ecotype) was crossed with wild-type (wt) Columbia ecotype (Col-0) or tip $\mathrm{KO}(\mathrm{Col}-0)$ plants. The F2 progeny derived from these crosses were genotyped for the presence of $H R T$ and tip. The HRT tip plants were morphologically similar to wt plants (Fig. 2A), did not show any visible or microscopic cell death (Fig. 2B), and showed basal expression of various defense genes (Fig. 2C). Upon TCV-inoculation, HRT tip plants developed HR within 3 days postinoculation (dpi), and the size and distribution of these lesions were similar to those in Di-17 or HRT TIP plants (Fig. 2A and B). The HR-forming leaves from $H R T$ tip plants also expressed increased levels of $P R-1, P R-2, P R-5$, and GST1 genes, similar to those in Di-17 or HRT TIP leaves (Fig. 2C).

The role of TIP in $H R T$-dependent HR formation was further evaluated in planta by analyzing $\mathrm{F} 1$ progeny derived out of a cross between Di-17 or HRT tip with plants overexpressing the TCV CP (35S-CP) (Cooley et al. 2000). Previously, we have shown that TCV CP acts as the avirulence factor, and plants expressing both $C P$ and $H R T$ show massive necrosis resulting in premature death at the seedling stage (Cooley et al. 2000). F1 seeds derived from Di- $17 \times 35 \mathrm{~S}-\mathrm{CP}$ or HRT tip $\times$ $35 \mathrm{~S}-C P$ crosses (16 each) were sown in soil, and all the F1 seeds showed massive necrosis resulting in eventual death of the F1 seeds (Fig. 2D). Together, these data suggest that a $\mathrm{KO}$ mutation in TIP does not impede HR formation or defensegene induction in response to TCV.

To determine if TIP was required for resistance to TCV, we studied the segregation of resistant plants in a TCV-inoculated F2 population derived from $\mathrm{Di}-17 \times \mathrm{Col}-0$ and $\mathrm{Di}-17 \times$ tip crosses (Table 1). Unlike HR, which cosegregates with $H R T$, resistance to TCV is dependent upon the presence of least one copy of HRT and a recessive locus, $r r t$ (Chandra-Shekara et al. 2004; Kachroo et al. 2000). Only HRT-containing plants showed resistance to $\mathrm{TCV}$, and approximately $25 \%$ of $\mathrm{F} 2$ plants from both crosses developed resistance (Table 1). Furthermore, approximately $25 \%$ of the HRT tip plants were resistant to TCV. This was further confirmed by RNA gel-blot analysis; HRT tip plants scored as susceptible showed presence of viral transcript in the systemic tissues (Fig. 2E). Further, the susceptible HRT tip accumulated a similar amount of viral transcript in the systemic tissues as the Col- 0 plants and showed typical stunted and crinkling phenotype (Fig. 2F). By comparison, the HRT tip scored as resistant did not show any viral transcript in the systemic tissues and developed normal
A
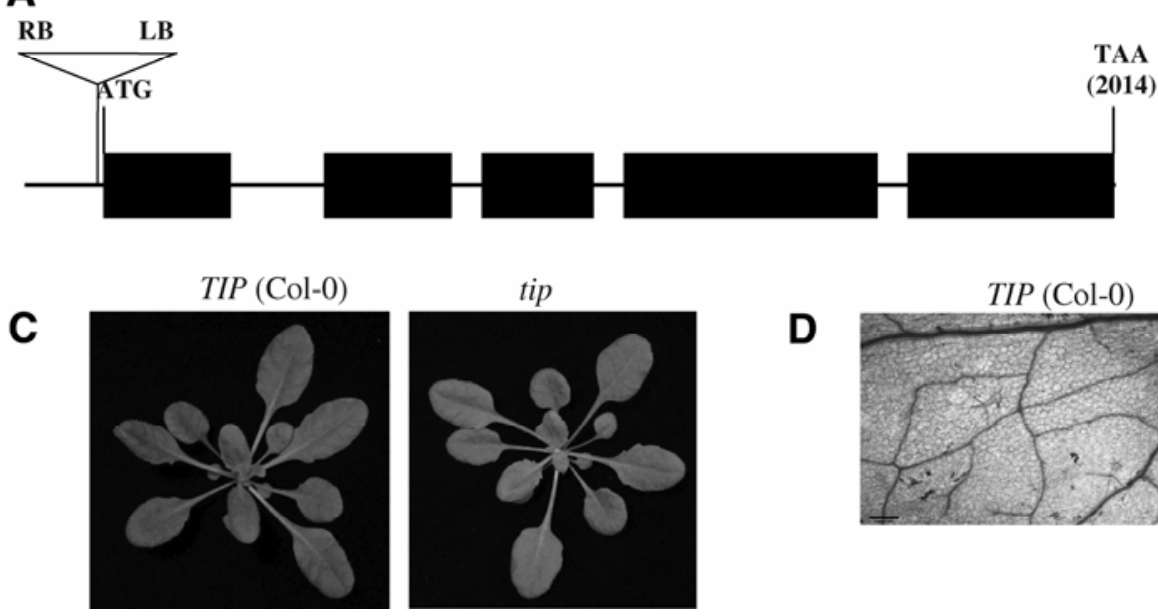

B

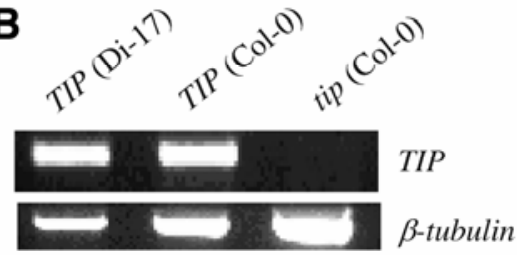

tip

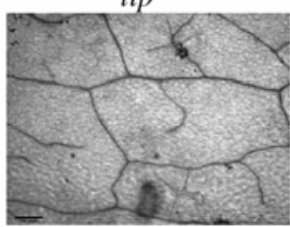

Fig. 1. Isolation of a knock-out (KO) mutation in TIP. A, Line diagram showing structure of the TIP gene and the site of T-DNA insertion. Black boxes represent exons and intervening lines represent introns. B, Reverse transcriptase-polymerase chain reaction analysis showing transcript levels of TIP in Di-17, Col- 0 , and the tip KO plants. The level of $\beta$-tubulin was used as an internal control to normalize the amount of cDNA template. C, Morphological phenotype of four-week-old Col-0 and tip plants. D, Microscopy of trypan blue-stained leaves from Col-0 and tip plants showing absence of any microscopic death in tip plants. 
bolts (Figs. 2E and F). Together, these data suggest that the null mutation in tip does not impair resistance to TCV.

\section{TIP is not required for SA-mediated induction of $H R T$.}

Exogenous application of SA or BTH upregulates $H R T$ expression and this increase in expression suppresses HR and enhances resistance to TCV in plants containing the $R R T$ allele

A
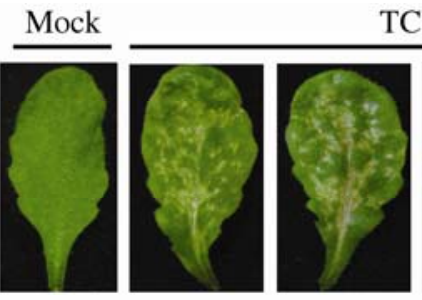

TCV

HRT tip TIP (Di-17) HRT tip HRT TIP TIP(Col-0)
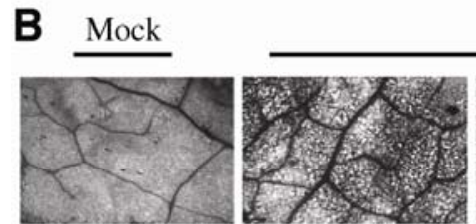

TCV

HRT tip

HRT tip

TIP (Col-0)

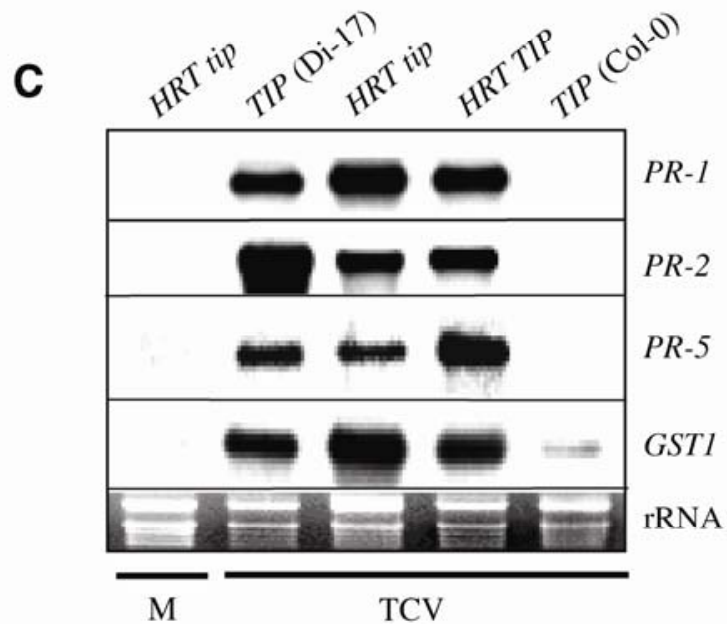

(Chandra-Shekara et al. 2004). We evaluated HRT tip plants for their abilities to induce SA-mediated HRT expression, suppress HR due to overexpression of $H R T$, or induce $H R T$-derived resistance in response to exogenous application of BTH. As expected, HR to TCV was suppressed in Di-17 plants pretreated with BTH 2 days prior to inoculation (Fig. 3A). This, in turn, correlated with increased levels of $H R T$ transcript in the
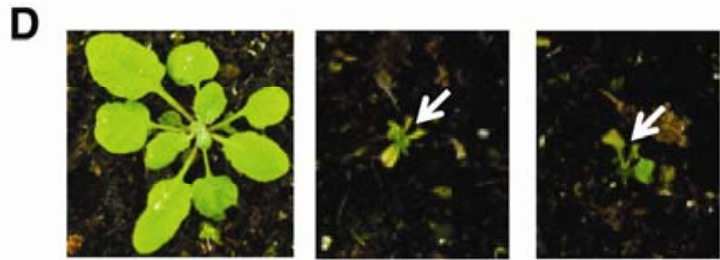

Di-17 x Col-0 Di-17 x 35S-CP HRT tip x 35S-CP

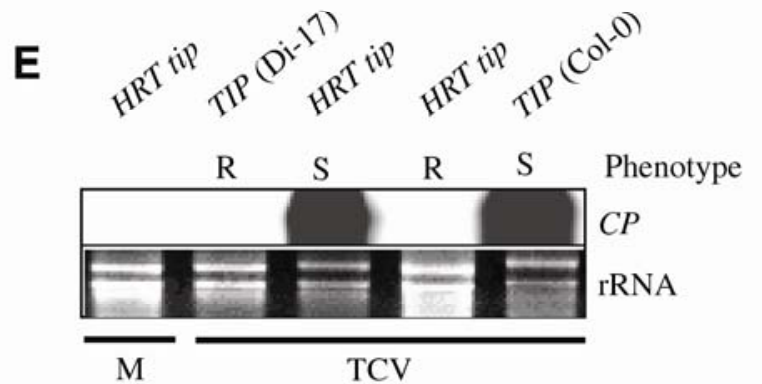

F

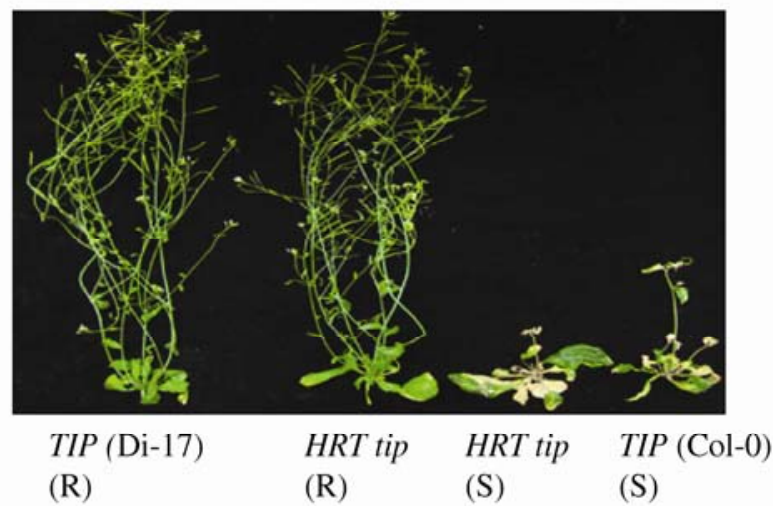

Fig. 2. Morphological and molecular phenotypes in HRT tip plants. A, Visible hypersensitive response formation in Turnip crinkle virus (TCV)-inoculated plants at 3 days postinoculation (dpi). All mock-inoculated plants showed absence of any visible lesions, similar to HRT tip. B, Microscopy of trypan bluestained leaves of mock- or TCV-inoculated plants. The Di-17 and HRT tip plants showed similar extent of cell death. C, Defense-gene expression in indicated genotypes at 3 days after mock- or TCV-inoculation. All mock-inoculated plants showed basal level expression of defense genes, similar to HRT tip. Ethidium bromide staining of rRNA was used as a loading control. D, Morphological phenotypes of two-week-old F1 plants derived from the indicated crosses. Arrow indicates F1 plants that remained stunted and eventually aborted. E, Systemic spread of TCV to uninoculated tissue in TCV-inoculated F2 plants derived from a Di-17 $\times$ tip cross. RNA was extracted from the uninoculated tissues at 18 dpi and was analyzed for the presence of the viral coat protein $(C P)$ transcripts. $\mathrm{R}$ and $\mathrm{S}$ indicate resistance and susceptible phenotypes, respectively. F, Typical morphological phenotypes of TCV-inoculated Di-17, Col-0, and a resistant and a susceptible HRT tip F2 plant derived from a Di-17 $\times$ tip cross. The susceptible plants showed crinkling, stunted bolt development, and drooping of bolts. Plants were photographed at $18 \mathrm{dpi}$.

Table 1. Segregation of resistance in Di- $17 \times$ Col- 0 and Di- $17 \times$ tip plants

\begin{tabular}{|c|c|c|c|c|c|c|c|c|}
\hline Cross $^{a}$ & Number of plants analyzed & Genotype $^{\text {b }}$ & Number of plants obtained & $\mathbf{H R}^{\mathrm{c}}$ & $\mathbf{R}^{\mathbf{d}}$ & $\mathbf{S}^{\mathbf{e}}$ & $\chi^{2}$ & $\mathbf{P}^{\mathbf{f}}$ \\
\hline Di- $17 \times$ Col- 0 & 148 & $H R T /-$ & 97 & + & 22 & 75 & 0.15 & 0.69 \\
\hline Di- $17 \times t i p$ & 394 & HRT/- tip/ tip & 47 & + & 9 & 38 & 0.85 & 0.35 \\
\hline & & HRT/- TIP/- & 183 & + & 40 & 143 & 0.96 & 0.32 \\
\hline
\end{tabular}

${ }^{a}$ The pollen-accepting plant is indicated first and the pollen donor second.

${ }^{\mathrm{b}}$ The genotype at $H R T$ and various mutant loci was determined by cleaved amplified polymorphic sequence analysis.

${ }^{\mathrm{c}} \mathrm{HR}=$ hypersensitive response.

${ }^{\mathrm{d}} \mathrm{R}=$ resistant, no disease symptoms.

${ }^{\text {e }} \mathrm{S}=$ susceptible, disease symptoms include crinkling of leaves and drooping of the bolt.

${ }^{\mathrm{f}}$ One degree of freedom. 
BTH-treated Di-17 plants (Fig. 4B). Similar to the Di-17 plants, exogenous application of BTH on HRT tip plants also suppressed HR and increased $H R T$ expression (Fig. 3A and B).

Next, we evaluated whether exogenous application of BTH altered susceptibility to TCV in HRT RRT tip plants. As expected, water-treated HRT RRT tip plants were susceptible and accumulated viral transcripts in their systemic tissues (Fig. 3C and D). In comparison, pretreatment with BTH induced resistance in approximately $65 \%$ of HRT RRT tip plants (Fig. 3C), and this was comparable to the BTH-induced resistance in $H R T$ $R R T$ or HRT RRT sid2 plants (Chandra-Shekara et al. 2004). Exogenous application of BTH on TIP or tip plants did not have any effect in the absence of $H R T$. Together, these results suggest that SA triggered increase in $H R T$ expression, suppression of HR, and enhanced resistance are independent of TIP.

The above results were further confirmed by mobilizing the tip mutation into the HRT ssi 2 background, which contains the $R R T$ allele and high endogenous SA (Kachroo et al. 2003,
2004). Previously, we have shown that the increased SA in the mutant ssi 2 background lead to increased $H R T$ expression and conferred resistance to TCV in an $R R T$-independent manner (Chandra-Shekara et al. 2004). The HRT ssi2 tip plants showed stunted morphology (data not shown) and constitutively expressed the $P R-1$ gene, similar to the HRT ssi2 plants (Fig. 4A). Unlike Di-17 and HRT tip, both HRT ssi2 and HRT ssi2 tip plants expressed high levels of HRT (Fig. 4B), which also correlated with enhanced resistance in these plants; approximately 95 to $98 \%$ of $H R T$ ssi2 and HRT ssi2 tip plants were resistant to TCV (Figs. 4C and D). Absence of ssi2 or HRT led to pronounced susceptibility. Analysis of viral transcripts detected high levels of TCV in the systemic tissues of susceptible genotypes (HRT tip, ssi2, ssi2 tip, and Col-0) but not in the resistant plants (Di-17, HRT ssi2, or HRT ssi2 tip) (Fig. 4E). Together, these results suggested that $T I P$ was neither required for the ssi2-induced increase in $H R T$ expression nor ssi2-conferred resistance in the HRT RRT background.
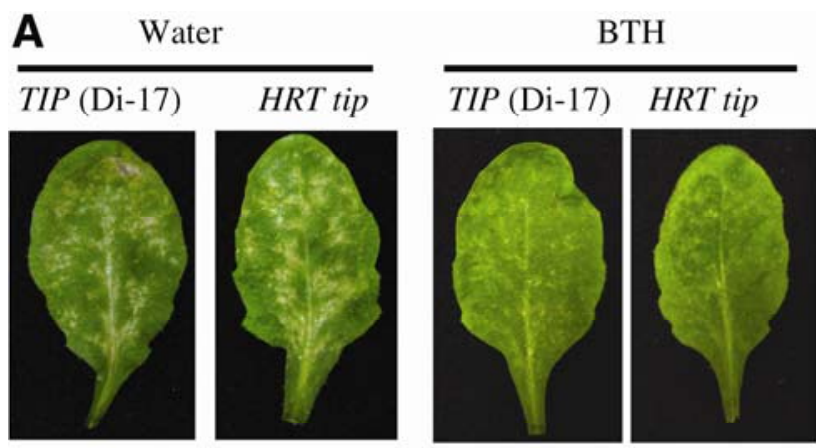

B
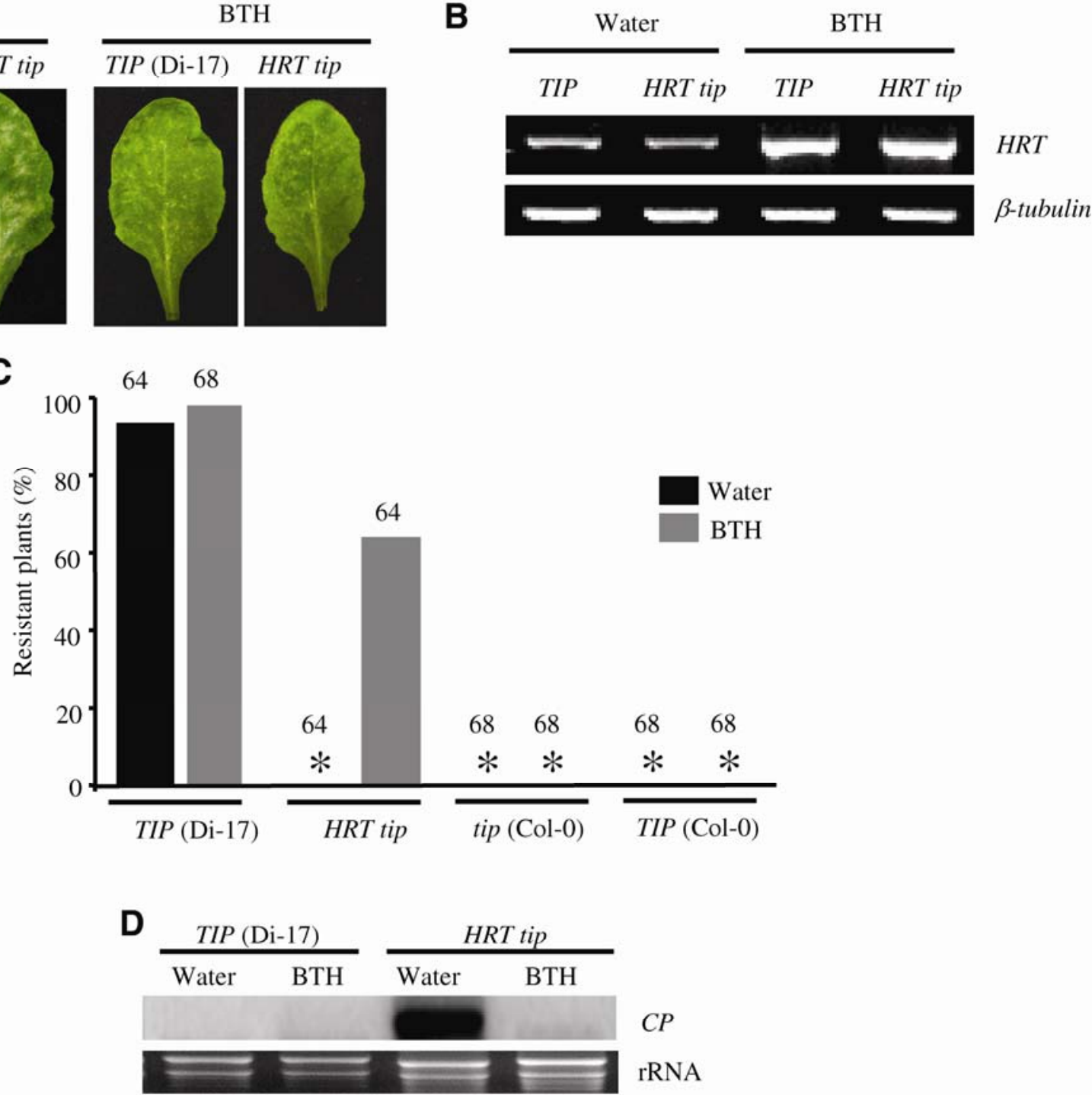

Fig. 3. Hypersensitive response (HR) formation, HRT transcript levels, Turnip crinkle virus (TCV) resistance, and systemic spread of TCV in HRT tip plants. A, Visible HR formation in water- and benzo (1,2,3) thiadiazole-7-carbothioic acid (BTH)-treated plants at 3 days postinoculation (dpi). B, Reverse transcriptase-polymerase chain reaction analyses showing expression of the $H R T$ gene in water- and BTH-treated plants. The level of $\beta$-tubulin was used as an internal control to normalize the amount of cDNA template. C, Percentage of TCV-resistant plants obtained after exogenous application of water or BTH. Resistance was analyzed 3 weeks postinoculation. The number of plants tested are indicated above each bar. The HRT tip plants are F3 progeny of a susceptible HRT tip F2 plant that was derived from a Di-17 × tip cross. HRT tip progeny from three different F3 lines were analyzed, and all showed similar results. Asterisk indicates $100 \%$ susceptibility. D, Systemic spread of TCV to uninoculated tissue in TCV-inoculated plants. RNA was extracted from the uninoculated tissues at $14 \mathrm{dpi}$ and was analyzed for the presence of the viral coat protein $(C P)$ transcripts. 
A mutation in TIP promotes replication of TCV and CMV.

Earlier reports showing interaction between CP and TIP (Ren et al. 2000, 2005) and our result that a null mutation in TIP does not impair HRT-mediated HR or resistance to TCV, suggested the possibility that TIP might serve as a host factor that regulates replication or movement, or both, of TCV in susceptible ecotypes like Col-0 and thus affect basal resistance. Therefore, TCV replication, as determined by CP levels, was assessed at 1,2, and $3 \mathrm{dpi}$ in inoculated leaves of TIP versus tip plants. There were no significant differences in accumulation of CP at 1 or 2 dpi (Fig. 5A). However, by 3 dpi tip plants consistently accumulated slightly increased levels of $\mathrm{CP}$ protein (Fig. 5A) and its corresponding transcript (data not shown). To assess if TIP plays a role in the systemic movement of TCV or appearance of disease symptoms, transcript levels of $C P$ (Fig. 5B) and symptoms (Fig. 5C) were analyzed in systemic tissues at 7 and $14 \mathrm{dpi}$. No differences were seen between wt and mutant plants. Therefore, TIP does not appear to play a significant role in symptom development to TCV.

To determine whether TIP functions in basal resistance to an unrelated viral pathogen, TIP and tip plants were inoculated with CMV and were assayed for CMV-CP levels in the inoculated leaves and disease symptoms at 1, 2, and 3 dpi. Strikingly, significantly higher levels of CMV CP were detected in tip versus TIP leaves throughout the timecourse (Fig. 5D). This correlated with enhanced disease symptoms; in comparison to TIP, tip plants showed severe stunting and drooping of the bolts (Fig. 5E). These data suggest that TIP is required for basal resistance to CMV.

To determine if TIP functions in basal- or $R$ gene-mediated resistance to a bacterial pathogen, TIP and tip plants were inoculated with virulent or avirulent (containing AvrRPT2) Pseudomonas syringae pv. tomato. No difference was detected between $T I P$ versus tip in their resistance status to either virulent or to avirulent $P$. syringae pv. tomato (Figs. 5F and G). Together, these data suggest that TIP is not required for basal- or RPS2-mediated resistance against $P$. syringae.

\section{DISCUSSION}

In this study, we have evaluated whether TIP plays a role in HRT-mediated HR and resistance to TCV. Earlier work carried
A

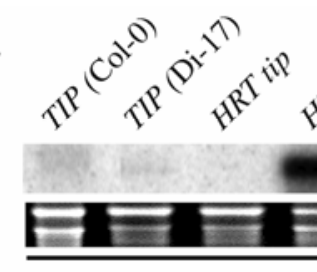

Mock

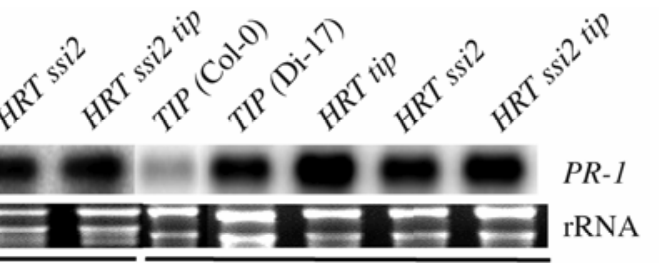

TCV
B

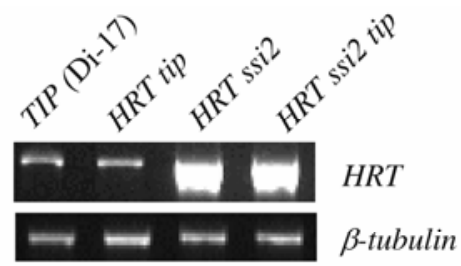

C

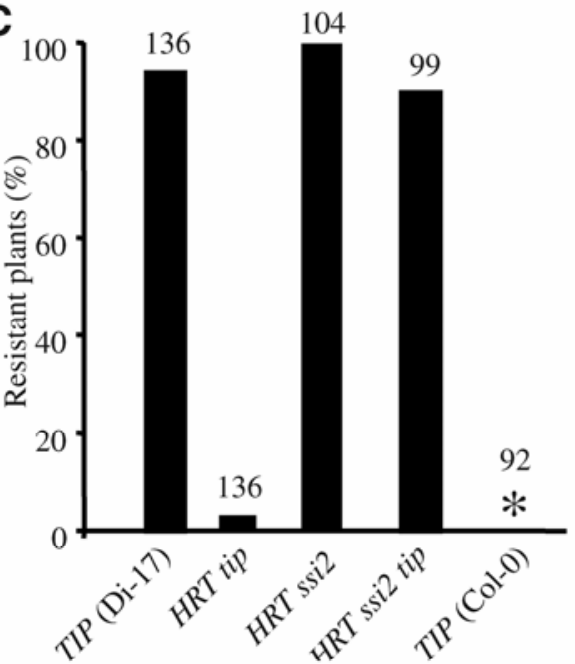

D

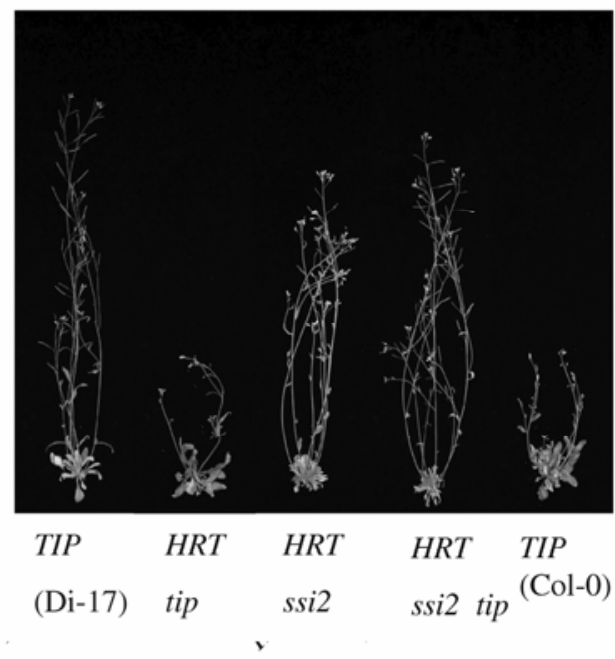

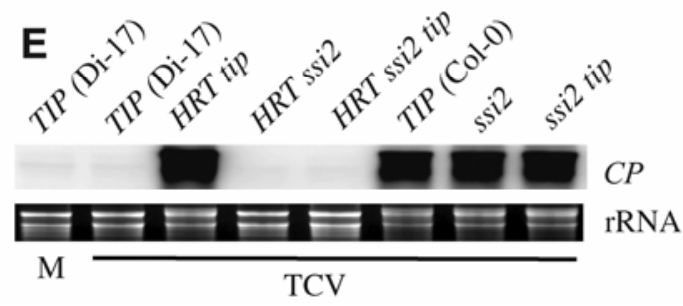

Fig. 4. Molecular analysis of HRT ssi2 tip plants. A, PR-1 gene expression in indicated genotypes at 3 days after mock- or Turnip crinkle virus (TCV)inoculation. Ethidium bromide staining of rRNA was used as a loading control. B, Reverse transcriptase-polymerase chain reaction analyses showing expression of HRT gene in TIP, HRT tip, HRT ssi2, and HRT ssi2 TIP plants. The level of $\beta$-tubulin was used as an internal control to normalize the amount of cDNA template. C, Percentage of TCV-resistant plants. Resistance was analyzed 3 weeks postinoculation. The numbers of plants tested are indicated above each bar. Asterisk indicates $100 \%$ susceptibility. D, Typical morphological phenotypes of TCV-inoculated plants at 14 days postinoculation (dpi). E, Systemic spread of TCV to uninoculated tissue of mock- or TCV-inoculated plants. RNA was extracted at 14 dpi and was analyzed for the presence of the viral coat protein $(C P)$ transcripts. 
out by Ren and associates (2005) showed that green fluorescent protein (GFP)-TIP was retained in the cytosol when coinfiltrated with TCV CP. This, together with the finding that TCV CP binds specifically to TIP, led to the assumption that the uneven distribution of TIP in the presence of CP signals the activation of HRT-mediated defense signaling (Ren et al. 2005). An alternate possibility is that HRT-mediated signaling was activated upon loss or degradation of TIP. Precedence for this is provided by the result that $P$. syringae avirulence protein AvrRPT2 induces degradation of the host-protein RIN4, and loss of RIN4 appears to activate RPS2-mediated resistance against $P$. syringae (Axtell and Staskawicz 2003; Mackey et al. 2003). Moreover, deletion of RIN4 is lethal in plants containing functional RPS2 but has no apparent phenotype in plants

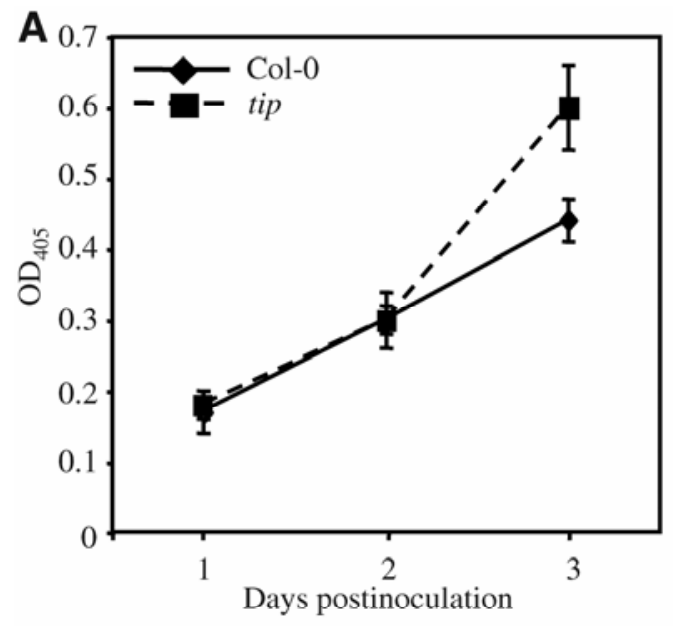

D

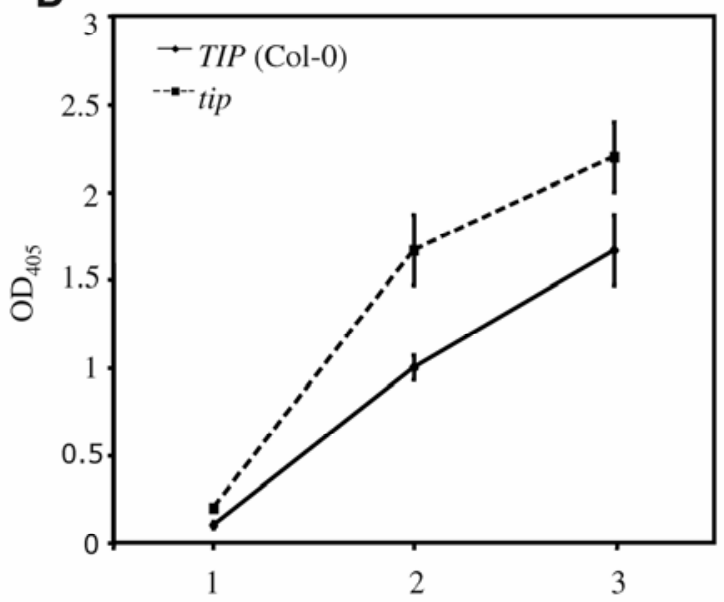

B

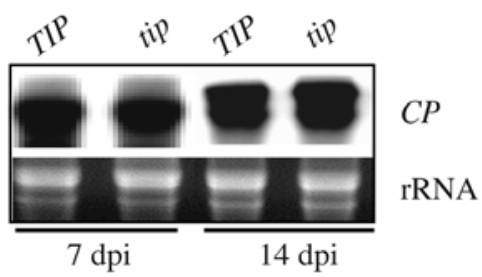

C

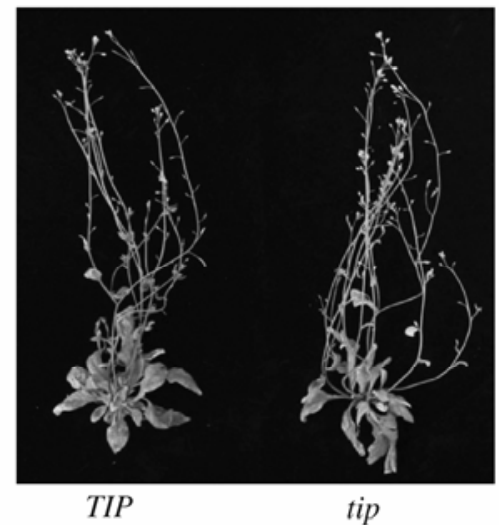

E

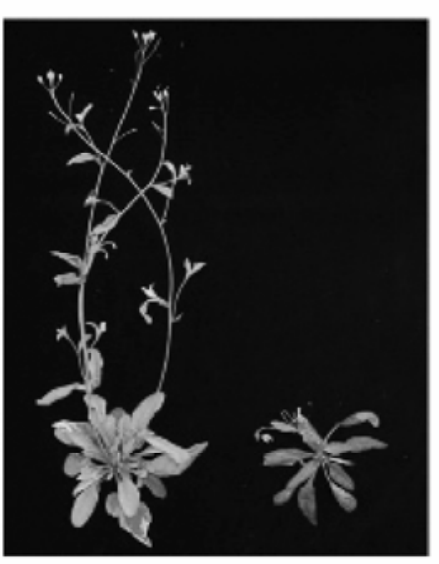

TIP (Col-0) tip
$\mathbf{F}$

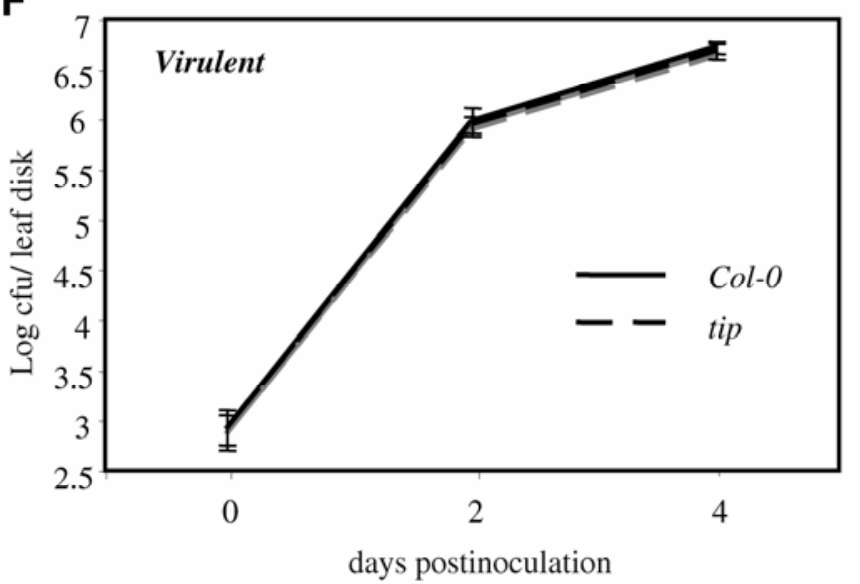

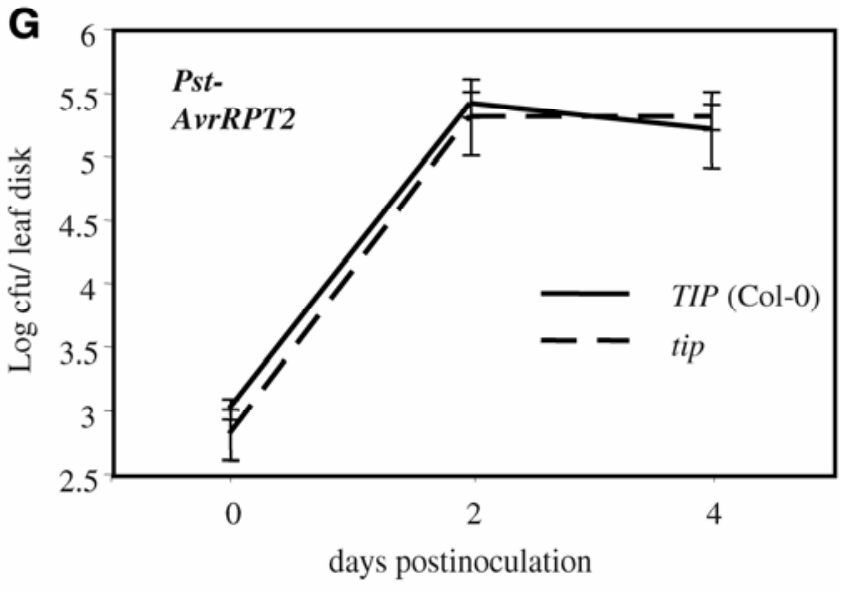

Fig. 5. Basal resistance in tip plants. A, Enzyme-linked immunosorbent assay (ELISA) showing levels of Turnip crinkle virus (TCV) coat protein (CP) in the inoculated leaves at 1 to 3 days postinoculation (dpi). B, Systemic spread of TCV to uninoculated tissue in TCV-inoculated plants. RNA was extracted from the uninoculated tissues at 7 and 14 dpi and was analyzed for the presence of the viral $C P$ transcripts. C, Typical morphological phenotypes of TCVinoculated plants at 14 dpi. D, ELISA showing levels of Cucumber mosaic virus (CMV) CP in the inoculated leaves at 1 to 3 dpi. E, Typical morphological phenotypes of CMV-inoculated plants at $10 \mathrm{dpi}$. F, Growth of a virulent isolate of Pseudomonas syringae on Col-0 and tip leaves. G, Growth of an avirulent isolate of P. syringae containing AvrRPT2 on Col-0 and tip leaves. 
lacking RPS2 (Axtell and Staskawicz 2003; Mackey et al. 2003). A similar possibility for the activation of HRT-mediated signaling can be ruled out, since HRT tip plants are wt-like and show basal expression of defense genes. Absence of detectable TIP transcript in tip mutant plants suggests that the $\mathrm{KO}$ mutation leads to a null phenotype. The normal HR and resistance in HRT tip plants suggest that these defense responses do not require a functional TIP. Therefore, we conclude that neither the binding between TIP and CP nor the cellular distribution of TIP contribute to HRT-mediated HR or resistance to TCV.

The possible role of TIP suggested by Ren and associates (2000, 2005) also relied upon the observation that mutations in $\mathrm{CP}$ that prevented their interaction with TIP abolished HR to TCV. A likely explanation for breakdown of resistance and HR seen upon inoculation of CP variants, which cannot bind TIP, is that HRT is unable to perceive the altered forms of $\mathrm{CP}$, which results in failure to activate HR and escape of the virus to the systemic tissues.

TIP is a member of the NAC family of proteins, which are involved in diverse physiological responses, ranging from development of plant embryos and flowers (Aida et al. 1997, 1999), lateral root development (Xie et al. 2000), cell division (Kim et al. 2006), defense (Collinge and Boller 2001; Xie et al. 1999), and abiotic stress responses (Fujita et al. 2004; Tran et al. 2004). The Arabidopsis genome contains 105 NAC-like transcription factors (Olsen et al. 2005; Ooka et al. 2003), which show varying levels of homology to TIP. It is, therefore, conceivable that TIP may fulfill redundant functions, and the loss of TIP could be compensated for by other TIP-like NAC proteins. Amino acid comparison and phylogenetic analysis of TIP to other members of the NAC family showed highest similarity to AtNTL6 (NAC62, Supplementary Fig. 1A). Furthermore, only AtNTL6 showed homology (approximately 60\% identity) to the C-terminal domain of TIP that was shown to interact with CP (Ren et al. 2005). However, two-hybrid analysis did not detect any interaction between the full-length $\mathrm{CP}$ and full-length or C-terminal 150 amino acids from AtNTL6 (data not shown), suggesting that AtNTL6 is unlikely to compensate for the loss of TIP.

A subclass of the NAC family of transcription factors contain a transmembrane domain at their $\mathrm{C}$-termini and are thereby membrane associated (Kim et al. 2007). The membrane-associated NAC proteins are usually expressed as dormant precursors, which upon controlled proteolytic cleavage are released from the membranes and enter into the nucleus to activate transcription of target genes. In a recent study conducted by Kim and associates (2007), the authors showed that overexpression of AtNTL6 (NAC62), which shows the highest similarity to TIP, produced a phenotype only if expressed as a C-terminal truncated protein. The $\mathrm{C}$-terminal truncation removed the transmembrane domain of AtNTL6, generating the active form of the transcription factor, which was now able to localize to the nucleus and initiate transcription. These results were further substantiated by immunolocalization studies, which showed that AtNTL6 localizes to the membranes and could be processed to a smaller molecular weight protein (Kim et al. 2007).

Structural analysis of TIP using the ARAMENNON membrane protein database predicts a strong $\propto$-helical transmembrane domain at the $\mathrm{C}$-terminal region. A comparison of amino acid sequence at the transmembrane domains of TIP and AtNTL6 showed approximately $80 \%$ identity, suggesting that, similar to AtNTL6, TIP may localize to membranes. However, the database prediction does not concur with the result that a GFP-TIP fusion protein localizes to the nucleus of tobacco cells (Ren et al. 2005). The altered localization of TIP in these experiments could either be the result of overexpression or misfolding due to presence of GFP.
A possible transmembranous nature of TIP and the fact that positive-sense viral replicase complexes are assembled on intracellular membranes (Ahlquist 2006) suggests the possibility that TIP may play a role in replication of CMV, TCV, or both. Increased levels of TCV and CMV CP in the inoculated leaves further support this possibility. Furthermore, the NAC family of transcription factors is known to modulate replication of Wheat dwarf geminivirus (Xie et al. 1999) and Tomato leaf curl virus (Selth et al. 2005). However, since viruses use different intercellular membranes for replication, the relationship between localization of TIP and viral replication remains unclear. Further examination of the requirement for TIP in CMV resistance could help elucidate the precise role of TIP in plant viral defense.

\section{MATERIALS AND METHODS}

\section{Plant growth conditions, pathogen infections, and genetic analysis.}

Plants were grown in the MTPS 144 Conviron walk-in chambers (Winnipeg, Canada) at $22^{\circ} \mathrm{C}$ with $65 \%$ relative humidity and a 14-h photoperiod. Transcripts synthesized in vitro from a cloned cDNA of TCV using T7 RNA polymerase were used for viral infections (Dempsey et al. 1993; Oh et al. 1995). For inoculations, the viral transcript was suspended in inoculation buffer at a concentration of $0.05 \mu \mathrm{g} / \mu \mathrm{l}$, and the inoculation was performed as described earlier (Dempsey et al. 1993). After viral inoculations, the plants were transferred to a Conviron MTR30 reach-in chamber maintained at $22^{\circ} \mathrm{C}$ with $65 \%$ relative humidity and a $14-\mathrm{h}$ photoperiod. Resistance and susceptibility were scored at 7 and 14 to $21 \mathrm{dpi}$ and was confirmed by Northern gel-blot analysis. Susceptible plants showed stunted growth, crinkling of leaves, and drooping of the bolt.

Inoculum for CMV strain Fny was prepared as described previously (Hu et al. 1998). Briefly, CMV was maintained on tobacco plants, and extract prepared from infected tobacco leaves was used as inoculum for Arabidopsis. Susceptible plants showed chlorosis on the inoculated leaves, stunted phenotypes, and drooping of the bolt.

Bacterial inoculations were carried out by injecting bacterial solution into the adaxial surface of leaves and two to three leaves were inoculated each plant. Four leaf disks were harvested from pathogen- or mock-inoculated leaves at 3 dpi and ground in $10 \mathrm{mM} \mathrm{MgCl}$, and the bacterial numbers were determined by titration. The bacterial numbers \pm standard deviation $(n=4)$ were presented as colony-forming units (CFU) per unit of leaf area $\left(25 \mathrm{~mm}^{2}\right)$. The experiment was independently performed twice with similar results.

Crosses were performed by pollinating flowers of Di-17 and HRT ssi2 plants with pollen from tip plants. The genotypes of the F2 plants at the TIP locus were determined by conducting polymerase chain reaction (PCR) analysis using left border and gene-specific primers (Fwd: GGTCCAAAGGACAAAA GA AGAG; Rev: CGAATTCTCAAAGTCTCACGC).

\section{Chemical treatment of plants.}

Three-week-old plants were sprayed or subirrigated with a solution of $500 \mu \mathrm{M}$ SA or $100 \mu \mathrm{M}$ BTH. Control plants were treated with water, and two days after treatment, three leaves per plant were inoculated with TCV RNA.

\section{RNA extraction and reverse transcriptase ( $R T)-P C R$ analysis.}

Small-scale RNA extractions was performed with TRIzol reagent (Invitrogen, Rockville, MD, U.S.A.), according to the manufacturer's instructions. RNA gel-blot analysis and synthesis of random primed probes were performed as described ear- 
lier (Kachroo et al. 2000). RT-PCR was performed using total RNA and HRT (Cooley et al. 2000) or TIP (ATGAAAGAAG ACATGGAAGTACTATC, CTCTTCTTTTGTCCTTTGGACC) gene-specific primers. Two to three independent RNA preparations were used for RT-PCR, and each of these were analyzed at least twice by RT-PCR. The RT-PCR was carried out for 35 cycles in order to determine absolute levels of transcripts. The number of amplification cycles was reduced to 23 to 25 in order to evaluate and quantify difference among transcript levels before they reached saturation.

\section{Enzyme-linked immunosorbent assay.}

Total protein was extracted in buffer containing $50 \mathrm{mM}$ Tris, $\mathrm{pH} 8.0,1 \mathrm{mM}$ EDTA, $12 \mathrm{mM} \beta$-mercaptoethanol, and $10 \mu \mathrm{g}$ of phenylmethylsulfonyl fluoride per milliliter. Proteins were fractionated on a 10 to $12 \%$ sodium dodecyl sulfate-polyacrylamide gel electrophoresis to confirm the quality. An antigencoated enzyme-linked immunosorbent assay was used to determine levels of TCV and CMV in the infected plants as described previously (Ghabrial and Schultz 1983).

\section{ACKNOWLEDGMENTS}

We thank S. Ghabrial for providing CMV isolate and anti-CMV coat protein antisera. We thank J. Morris for providing anti-TCV CP antisera. We thank L. Lapchyk for technical expertise and A. Crume for help with managing plant growth facility. We thank the Arabidopsis Biological Resource Center database for SALK_137395 seeds. This work was supported by grants from the National Science Foundation (IOS-0641576) and the Kentucky Science and Engineering Foundation (4-67835). This study is publication No. 08-12-087 of the Kentucky Agricultural Experiment Station.

\section{LITERATURE CITED}

Aida, M., Ishida, T., Fukaki, H., Fujisawa, H., and Tasaka, M. 1997. Genes involved in organ separation in Arabidopsis: An analysis of the cupshaped cotyledon mutant. Plant Cell 6:841-857.

Aida, M., Ishida, T., and Tasaka, M. 1999. Shoot apical meristem and cotyledon formation during Arabidopsis embryogenesis interaction among the cup-shaped cotyledon and shoot meristemless genes. Development 126:1563-1570.

Ahlquist, P. 2006. Parallels among positive-strand RNA viruses, reversetranscribing viruses and double-stranded RNA viruses. Nature Rev. Microbiol. 4:371-382.

Axtell, M. J., and Staskawicz, B. J. 2003. Initiation of RPS2-specified disease resistance in Arabidopsis is coupled to the AvrRpt2-directed elimination of RIN4. Cell 112:369-377.

Bittner-Eddy, P. D., and Beynon, J. L. 2001. The Arabidopsis downy mildew resistance gene, $R P P 13-N d$, functions independently of $N D R 1$ and EDS1 and does not require the accumulation of salicylic acid. Mol. Plant-Microbe Interact. 14:416-421.

Century, K. S., Holub, E. B., and Staskawicz, B. J. 1995. NDR1, a locus of Arabidopsis thaliana that is required for disease resistance to both a bacterial and a fungal pathogen. Proc. Natl. Acad. Sci. U.S.A. 92:65976601.

Chandra-Shekara, A. C., Navarre, D., Kachroo, A., Kang, H. G., Klessig, D., and Kachroo, P. 2004. Signaling requirements and role of salicylic acid in $H R T$ - and rrt-mediated resistance to Turnip crinkle virus in Arabidopsis. Plant J. 5:647-59.

Chandra-Shekara, A. C., Gupte, M., Navarre, D., Raina, S., Raina, R., Klessig, D., and Kachroo, P. 2006. Light-dependent hypersensitive response and resistance signaling to Turnip crinkle virus in Arabidopsis. Plant J. 45:320-334

Collinge, M., and Boller, T. 2001. Differential induction of two potato genes, Stprx 2 and StNAC, in response to infection by Phytophthora infestans and to wounding. Plant Mol. Biol. 46:521-529.

Cooley, M. B., Pathirana, S., Wu, H. J., Kachroo, P., and Klessig, D. F. 2000. Members of the Arabidopsis HRT/RPP8 family of resistance genes confer resistance to both viral and oomycete pathogens. Plant Cell 12:663-676.

Dempsey, D. A., Wobbe, K. K., and Klessig, D. F. 1993. Resistance and susceptible responses of Arabidopsis thaliana to turnip crinkle virus. Phytopathology 83:1021-1029.

Flor, H. 1971. Current status of gene-for-gene concept. Annu. Rev. Phytopathol. 9:275-296.
Fujita, M., Fujita, Y., Maruyama, K., Seki, M., Hiratsu, K., Ohme-Takagi, M., Tran, L. S., Yamaguchi-Shinozaki, K., and Shinozaki, K. 2004. A dehydration-induced NAC protein, RD26, is involved in a novel ABAdependent stress-signaling pathway. Plant J. 39:863-876.

Ghabrial, S. A., and Schultz, F. G. 1983. Serological detection of bean pod mottle virus in bean leaf beetles. Phytopathology 73:480-483.

Hu, C. C., Sanger, M., and Ghabrial, S. A. 1998. Production of infectious RNA transcripts from full-length cDNA clones representing two subgroups of peanut stunt virus strains: Mapping satellite RNA support to RNA1. J. Gen. Virol. 79:2013-2021.

Innes, R. 2004. Guarding the goods. New insights into the central alarm system of plants. Plant Physiol. 135:695-701.

Jia, Y., McAdams, S. A., Bryan, G. T., Hershey, H. P., and Valent, B. 2000. Direct interaction of resistance gene and avirulence gene products confers rice blast resistance. EMBO (Eur. Mol. Biol. Organ.) J. 19:40044014.

Kachroo, A., Lapchyk, L., Fukushigae, H., Hildebrand, D., Klessig, D., and Kachroo, P. 2003. Plastidial acid signaling modulates SA- and JA-mediated signaling in the Arabidopsis ssi2 mutant. Plant cell 15:2952-2965.

Kachroo, A., Srivatha C. V., Lapchyk, L., Falcone, D., Hildebrand, D., and Kachroo, P. 2004. Oleic acid levels regulated by glycerolipid metabolism modulate defense gene expression in Arabidopsis. Proc. Natl. Acad. Sci. U.S.A. 101:5152-5157.

Kachroo, P., Yoshioka, K., Shah, J., Dooner, H. K., and Klessig, D. F. 2000. Resistance to Turnip crinkle virus in Arabidopsis is regulated by two host genes, is salicylic acid dependent but NPR-1, ethylene and jasmonate independent. Plant Cell 12:677-690.

Kachroo, P., Chandra-Shekara, A. C., and Klessig, D. 2006. Plant signal transduction and defense against viral pathogens. Advances in Viral Research. 66:161-191.

Kim, S. Y., Kim, S. G., Kim, Y. S., Seo, P. J., Bae, M., Yoon, H. K., and Park, C. M. 2007. Exploring membrane-associated NAC transcription factors in Arabidopsis: Implications for membrane biology in genome regulation. Nucleic Acids Res. 35:203-213.

Kim, Y. S., Kim, S. G., Park, J. E., Park, H. Y., Lim, M. H., Chua, N. H., and Park, C. M. 2006. A membrane-bound NAC transcription factor regulates cell division in Arabidopsis. Plant Cell 18:3132-3144.

Leister, R. T., and Katagiri, F. 2000. A resistance gene product of the nucleotide binding site- leucine rich repeats class can form a complex with bacterial avirulence proteins in vivo. Plant J. 22:345-354.

Mackey, D., Holt III, B. F., Wiig, A., and Dangl, J. L. 2002. RIN4 interacts with Pseudomonas syringae type III effector molecule and is required for RPM1-mediated resistance in Arabidopsis. Cell 108:743-754.

Mackey, D., Belkhadir, Y., Alonso, J. M., Ecker, J. R., and Dangl, J. L. 2003. Arabidopsis RIN4 is a target of the type III virulence effector AvrPrt2 and modulates RPS2-mediated resistance. Cell 112:379-389.

McDowell, J. M., Cuzick, A., Can, C., Beynon, J., Dangl, J. L., and Holub, E. B. 2000. Downy mildew (Peronospora parasitica) resistance genes in Arabidopsis vary in functional requirements for NDR1, EDS1, NPR1 and salicylic acid accumulation. Plant J. 22:523-29.

Nimchuk, Z., Marois, E., Kjemtrup, S., Leister, R. T., Katagiri, F., and Dangl, J. L. 2000. Eukaryotic fatty acylation drives plasma membrane targeting and enhances function of several type III effector proteins from Pseudomonas syringae. Cell 101:353-363.

Oh, J. W., Kong, W., Song, C., Carpenter, C. D., and Simon, A. E. 1995. Open reading frames of Turnip crinkle virus involved in satellite symptom expression and incompatibility with Arabidopsis thaliana ecotype Dijon. Mol. Plant-Microbe Interact. 8:979-987.

Olsen, A. N., Ernst, H. A., Leggio, L. L., and Skriver, K. 2005. NAC transcription factors: Structurally distinct, functionally diverse. Trends Plant Sci. 10:79-87.

Ooka, H., satoh, K., Nagato, T., Otoma, Y., Murakami, K., Matsubara, K. Osato, N., Kawai, J., Carninci, P., Hayashizaki, Y., Suzuki, K., Kojima, K., Takahara, Y., Yamamoto, K., and Kikuchi, S. 2003. Comprehensive analysis of NAC family genes in Oryza sativa and Arabidopsis thaliana. DNA Res. 10:239-247.

Parker, J. E., Houlb, E. B., Frost, L. M., Falk, A., Gunn, N. D., and Daniels, M. J. 1996. Characterization of eds1, a mutation in Arabidopsis suppressing resistance to Pernospora parasitica specified by several different RRP genes. Plant Cell 8:2033-2046.

Ren, T., Qu, F., and Morris, T. J. 2000. HRT gene function requires interaction between a NAC protein and viral capsid protein to confer resistance to Turnip crinkle virus. Plant Cell 12:1917-1926.

Ren, T., Qu, F., and Morris, T. J. 2005. The nuclear localization of the Arabidopsis transcription factor TIP is blocked by its interaction with the coat protein of Turnip crinkle virus. Virology 331:316-324.

Rooney, H. C. E., van't Klooster, J. W., van der Hoorn, R. A. L., Joosten, M .H .A. J., Jones, J. D. G., and de Wit, P. J. G. M. 2005. Cladosporium Avr2 inhibits tomato Rcr3 protease required for $C f$-2-dependent disease resistance. Science 308:1783-1786. 
Scofield, S. R., Tobias, C. M., Rathjen, J. P., Chang, J. H., Lavelle, D. T. Michelmore, R. W., and Staskawicz, B. J. 1996. Molecular basis of gene-for-gene specificity in bacterial speck disease of tomato. Science 274:2063-2065.

Selth, L. A., Dogra, S. C., Rasheed, M. S., Healy, H., randles, J. W., and Rezaian, M. A. 2005. A NAC domain protein interacts with tomato leaf curl virus replication accessory protein and enhances viral replication. Plant Cell 17:311-325.

Shao, F., Golstein, C., Ade, J., Stoutemyer, M., Dixon, J. E., and Innes, R. W. 2003. Cleavage of Arabidopsis PBS1 by a bacterial type III effector. Science 301:1230-1233.

Soosaar, J. L., Burch-Smith, T. M., and Dinesh-Kumar, S. P. 2005. Mechanisms of plant resistance to viruses. Nat. Rev. Microbiol. 3(10):789798

Tang, X., Frederick, R. D., Zhou, J., Halterman, D. A., Jia, Y., and Martin, G. B. 1996. Initiation of plant disease resistance by physical interaction of AvrPto and Pto kinase. Science 274:2060-2063.

Tran, L. S., Nakashima, K., Sakuma, Y., Simpson, S. D., Fujita, Y.,
Maruyama, K., Fujita, M., Seki, M., Shinozaki, K., and YamaguchiShinozaki, K. 2004. Isolation and functional analysis of Arabidopsis stress-inducible NAC transcription factors that bind to a drought-responsive cis-element in the early responsive to dehydration stress 1 promoter. Plant Cell 16:2481-2498.

Van der Biezen, E. A., and Jones, J. D. 1998. Plant disease-resistance proteins and the gene-for-gene concept. Trends Biochem. Sci. 23:454-456.

Xie, Q., Frugis, G., Colgan, D., and Chua, N. H. 2000. Arabidopsis NAC transduces auxin signal downstream of TIR1 to promote lateral root development. Genes Dev. 14:3024-3036.

Xie, Q., Sanz-Burgos, A. P., Guo, H., Garcia, J. A., and Gutierrez, C. 1999. GRAB proteins, novel members of the NAC domain family, isolated by their interaction with a geminivirus protein. Plant Mol. Biol. 39:647656.

Zhao, Y., DelGrosso, L., Yigit, E., Dempsey, D. A., Klessig, D. F., and Wobbe, K. K. 2000. The amino terminus of the coat protein of Turnip crinkle virus is the AVR factor recognized by resistant Arabidopsis. Mol. Plant-Microbe Interact. 13:1015-1018. 